\title{
Probabilistic analyses of tunneling-induced ground movements
}

\author{
Guilhem Mollon - Daniel Dias • Abdul-Hamid Soubra
}

Received: 9 November 2011/ Accepted: 20 July 2012

(C) Springer-Verlag 2012

\begin{abstract}
Tunneling-induced ground movements are investigated in this paper using both deterministic and probabilistic analyses. The deterministic model is based on three-dimensional (3D) numerical simulations using the commercial code FLAC $^{3 \mathrm{D}}$. This model attempts to reproduce some major phenomena during a typical slurryshield tunnel excavation (ground movements due to the applied face pressure, the overcutting, the shield conicity, the annular void behind the shield, and the grout injection in this void). Moreover, the model provides useful information about the nature and magnitude of the soil movements at the ground surface. A probabilistic study is then undertaken in order to evaluate the impact of the variability of several input variables on the ground movements. An efficient probabilistic method called CSRSM is used to assess this uncertainty propagation. In a last section, the output variables of the model are linked to failure criteria. This allows one to determine probabilities of failure, depending on the probabilistic properties of the input variables and on the admissible threshold of each criterion.
\end{abstract}

G. Mollon ( $\square)$

Department of Civil and Environmental Engineering, HKUST, Clear Water Bay, Kowloon, Hong Kong

e-mail: guilhem.mollon@gmail.com

D. Dias

Joseph Fourier University, LTHE UMR 5564, Grenoble, France e-mail: daniel.dias@ujf-grenoble.fr

A.-H. Soubra

University of Nantes, Saint-Nazaire, France

e-mail: abed.soubra@univ-nantes.fr
Keywords CSRSM · Ground movements · Failure probability $\cdot$ Settlements $\cdot$ Tunnel

\section{Introduction}

Two main issues may occur during the excavation of a shallow tunnel driven by a pressurized shield. The first one is related to a possible instability of the pressurized face, and the second one is related to ground movements around the excavation. The first problem is solved by the application of a retaining fluid pressure on the tunnel face, and the value of this pressure may be computed with analytical or numerical models $[3,14,15,18,20,22,23$, $33]$. The second problem has also been studied by several authors $[2,4-6,11,12,19,21,26,27,30]$ and it will be investigated in depth in the present paper. In the case of a pressurized tunnel excavation, a number of modern technical solutions which aim at limiting the perturbations of the existing at rest soil pressures are generally used in practice. Among these solutions, one may cite (a) the application of a retaining face pressure, (b) the use of a cylindrical shield behind the cutting wheel, (c) the installation of concrete lining under the protection of this shield, and (d) the injection of a highly pressurized grout behind the shield to fill the void between the lining and the excavated soil. Despite these technical solutions, a tunnel excavation is never "neutral" with respect to the excavated soil, and causes inevitably disturbance to the surrounding soil. These perturbations lead to soil displacements around the excavation (e.g., settlements or horizontal displacements), which propagate to the surface and may have important impacts on existing structures. Because of the complexity of the technical solutions used to limit these perturbations, the modeling of these 
phenomena is not an easy task. Several authors proposed analytical or numerical models for the prediction of soil displacements. The early numerical models were based on plane strain or axisymmetric assumptions. Apparently, three dimensional analyses are more appropriate [4-6, 11, $12,19,21,26,27,30]$. Besides, when trying to predict the soil movements, the following uncertainties should be considered: (a) the natural variability of the soil, (b) the possible errors or omissions in the measurements of the soil characteristics, or (c) the imperfect control of the tunnel boring machine. The first aim of this paper is to propose a numerical model that is able to predict the soil movements in the neighborhood of a pressurized tunnel excavation. In a second part, the uncertainties of several input parameters are mathematically modeled by random variables, and an efficient probabilistic method called CSRSM is used to evaluate the propagation of the uncertainty from the input to the output variables of the deterministic model. This makes possible a complete statistical description of the variability of the soil movements induced by the excavation. Finally, the definition of rigorous criteria of admissible soil movements allows one to assess the probability of failure of a tunnel excavation driven by a pressurized shield.

\section{Deterministic model}

In a previous study by the same authors ([21]), a simplified numerical model for the computation of the ground movements induced by a pressurized shield was presented. This model computed the soil movements due to the applied face pressure (which may be smaller or greater than the existing at rest pressures), and it did not take into account the others processes during the tunnel excavation which can induce volume loss.

The aim of the proposed numerical model by FLAC ${ }^{3 \mathrm{D}}$ is to take into account a large number of phenomena which take place during the excavation of the tunnel (soil movements due to the applied face pressure, the overcutting, the shield conicity, the annular void behind the shield, and the grout injection in this void). For this reason, the geometry of the 3D model is designed to be as close as possible to the actual geometry of such an excavation. This model is not based on a real case, but it aims at representing in their generality the common features of the excavation method. If needed, its dimensions may be modified to fit with the ones of a real project. Thus, this paper focuses on a general parametric study and the dimensions of the model are chosen arbitrarily.

A schematic view of the tunnel boring machine (TBM) in the vertical plane of symmetry of the tunnel is provided in Fig. 1. A slurry-shield TBM of diameter $D=10 \mathrm{~m}$, under a cover depth $C=10 \mathrm{~m}$ was considered in the analysis. This geometry $(C=D)$ corresponds to a very shallow tunnel. It is chosen herein since it is the most critical case that may be encountered in the design of real projects and it leads to the biggest ground movements. The rock bed (which is taken as the lower boundary of the numerical model) is assumed to be located $10 \mathrm{~m}$ below the tunnel invert. On the other hand, the groundwater table is assumed to be located below the invert of the tunnel. Thus, it has limited effect on the ground movements and is neglected in the study. Notice that in the remainder of this article, the term "upstream" will refer to the areas located behind the shield (with respect to the excavation direction), while the term "downstream" will refer to the areas that have not been excavated yet.

The face pressure presents a trapezoidal profile to account for the density of the slurry. The unit weight is $12 \mathrm{kN} / \mathrm{m}^{3}$. The value of the average pressure (applied at the tunnel axis) is called $\sigma_{t}$. The pressure diagrams applied to the circular tunnel face and to the cylindrical surface just behind it are shown in Fig. 1. The cylindrical surface is $1 \mathrm{~m}$ long. It corresponds to the excavation chamber situated between the excavated face and the shield. In order to account for the slight overcutting that can be observed in real cases, the maximal radius of the shield is $1.5 \mathrm{~cm}$ shorter than the one of the excavated tunnel (cf. Fig. 1). This space may allow a possible migration of the slurry on a short distance behind the cutting wheel. This slurry is simulated by a triangular pressure diagram on a length of $1 \mathrm{~m}$. Besides, the shield conicity is taken into account in the analysis. The backside radius of the shield is thus $1 \mathrm{~cm}$ shorter than the foreside one. The total length of the shield is $11 \mathrm{~m}$. For the sake of simplicity, the shield is not introduced in the numerical model by means of a set of volume elements like the surrounding soil. Instead, it is replaced by a condition on the soil displacements which states that the soil cannot "penetrate" into the shield (even though this shield does not exist in the model). Thus, at each excavation stage, the position of the envelope of the "fictitious" shield is calculated and each node of the soil located on the contour of this virtual shield is artificially blocked. Behind the shield, a grout is injected under pressure to avoid a possible soil decompression related to the annular void appearing between the excavated soil and the concrete lining (which is positioned under the shield during its progression). The concrete lining is supposed to be perfectly rigid. This assumption is actually not exact in reality since the lining is always made of deformable materials, but this deformability is generally known to have a limited effect on the ground movements when compared to other phenomena (such as face decompression or grout consolidation). Besides, another simplified 


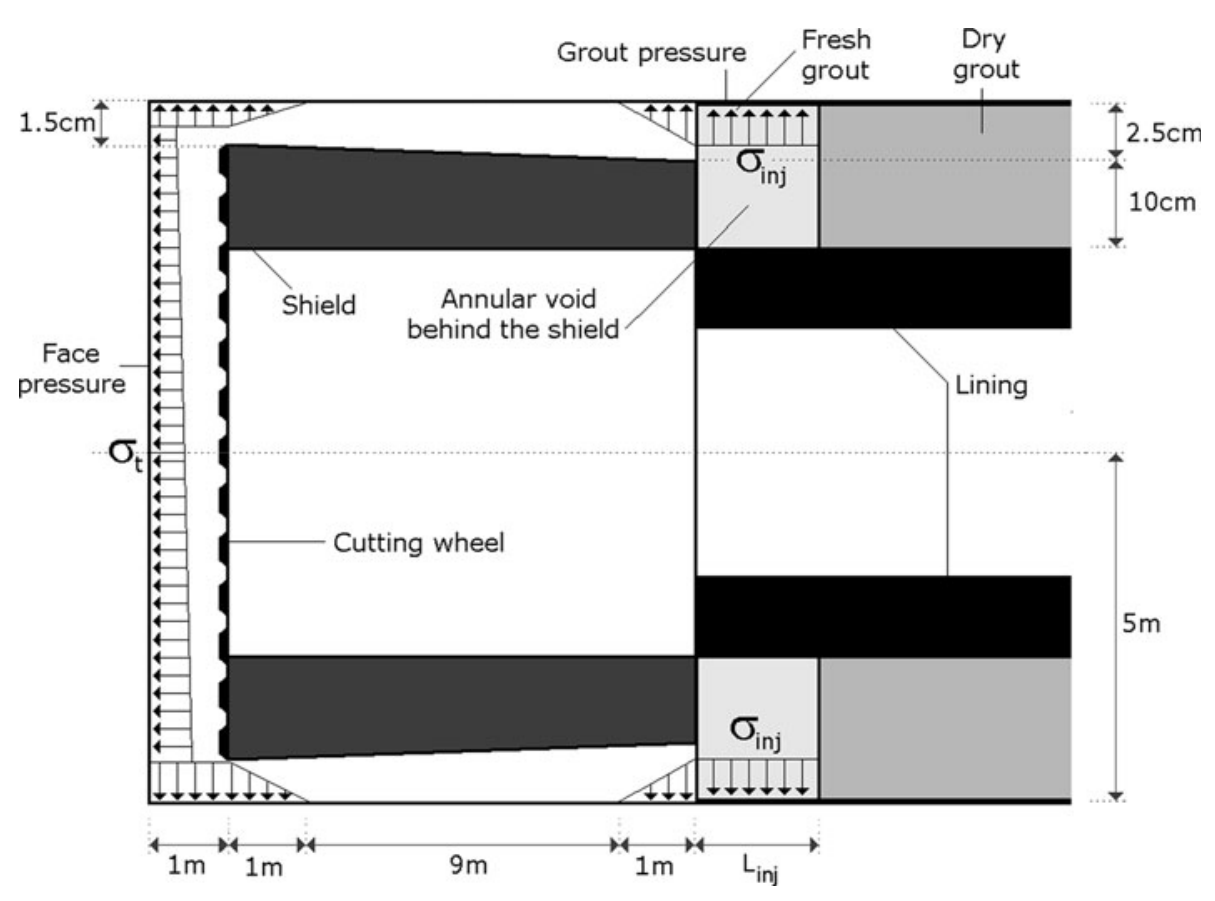

Fig. 1 Layout of the TBM

assumption related to the perfect contact between the lining and the surrounding soil is adopted in this study. This is because the interface elements at this contact area have led to non-convergent solutions.

The injected grout progressively hardens from a liquid to a solid state during the TBM progression. To simulate this phenomenon, it is considered that the grout is liquid on a length $L_{i n j}$ behind the shield (in this case, the grout is simulated by a uniform pressure $\sigma_{i n j}$ applied to the cylindrical surface of the excavated soil) and solid beyond $L_{i n j}$ (in this case, the grout is simulated by volume elements with a perfect elastic behavior, with the elastic characteristics $E_{\text {grout }}=10 \mathrm{MPa}$ and $v_{\text {grout }}=0.22$ ). The length $L_{i n j}$ is fixed to $3 \mathrm{~m}$ as a first rough estimate. Obviously, this is a very simplified way to simulate the grout consolidation and solidification. However, it was chosen herein for its simplicity, for its low computational cost in a probabilistic framework and because it has already been used with success in [27].

As for the case of the face pressure, the annular void existing between the backside of the shield and the excavated soil makes possible the migration of some grout toward the shield. This migration is simulated by a triangular pressure on a length of $1 \mathrm{~m}$ as shown in Fig. 1.

The 3D numerical model used in FLAC ${ }^{3 \mathrm{D}}$ is shown in Fig. 2. It is composed of approximately 98,000 zones. The length of an excavation step is $1 \mathrm{~m}$, and 35 excavation steps are performed successively. This number of excavation steps is not arbitrarily chosen and will be explained later in this paper. The excavation is performed along the $Y$ axis, $X$ and $Z$ being the transversal and the vertical directions, respectively. The constitutive model for the soil is an elastic perfectly plastic model with a Mohr-Coulomb failure criterion and a non-associated flow rule. Its parameters are set to the following illustrative values: $E=50 \mathrm{MPa}, v=0.3, c=7 \mathrm{kPa}, \varphi=17^{\circ}$, and $\psi=0^{\circ}$ (dilatancy angle). The soil unit weight is taken equal to $\gamma=18 \mathrm{kN} / \mathrm{m}^{3}$. It is well known that an elastic perfectly plastic constitutive model with a Mohr-Coulomb failure criterion is not able to accurately reproduce the soil movements induced by excavation, but it was chosen herein for the sake of simplicity. In fact, this model aims at showing the merits of the probabilistic method presented later in this paper.

The common method to choose the values of the face and grout pressures makes use of the vertical stress $\sigma_{v}$ in the soil mass before excavation. The face pressure is thus often set to:

$\sigma_{t} \approx K_{0} \cdot \sigma_{v}$

where $K_{0}$ is the at rest earth pressure coefficient. It can be computed by the classical Jaky formula $\left(K_{0}=1-\sin \varphi\right)$, and $\sigma_{v}$ is the soil overburden pressure at the tunnel axis (i.e., at a depth of $15 \mathrm{~m}$ in the present case). Notice that the use of Eq. 1 is not sufficient to achieve immediate equilibrium of the tunnel face, since the self-weights of the soil and of the slurry are not equal. Thus, the horizontal stress may be perfectly balanced at the center of the tunnel face, but this is not the case at the crown and at the foot of 


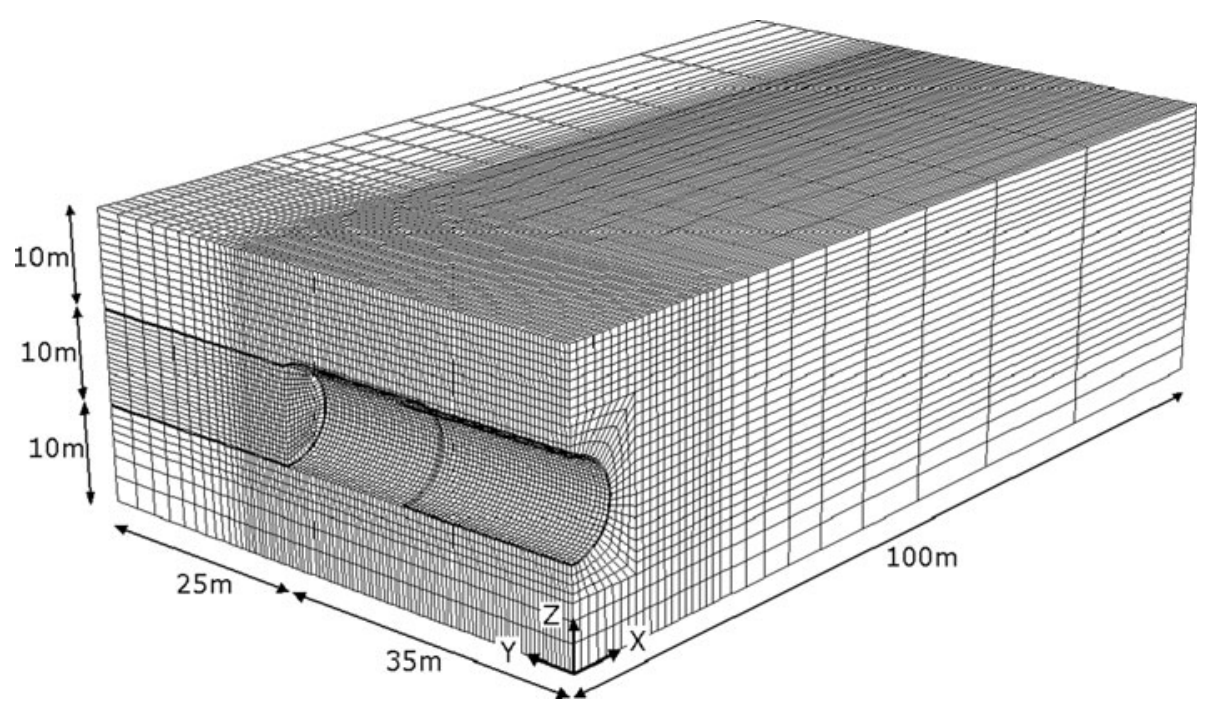

Fig. 2 Perspective view of the numerical model introduced in FLAC ${ }^{3 D}$

the face. Moreover, the value of $K_{0}$ cannot be determined exactly in practice. The grout pressure applied to the tail void is often set to:

$\sigma_{i n j} \approx 1.2 \cdot \sigma_{v}$

In this expression, $\sigma_{v}$ is the soil overburden pressure at the tunnel crown, that is, at a depth of $10 \mathrm{~m}$ in the present case. For the soil parameters and the geometry chosen for the model, the applied pressures considered in the present analysis are taken equal to $\sigma_{t}=190 \mathrm{kPa}$ and $\sigma_{i n j}=215 \mathrm{kPa}$.

\section{Deterministic results}

\subsection{Settlements}

Figure 3 presents the vertical displacement and the horizontal displacement (in the $Y$ direction) along the $Y$ axis for the points located on the ground surface as obtained after 35 excavation stages. Each point of this figure corresponds to a point of the mesh introduced in FLAC ${ }^{3 \mathrm{D}}$. In the case of a real excavation in homogeneous soil, constant displacements are expected to occur behind the tunnel face due to a stabilization of the soil after the TBM pass. On Fig. 3, however, the settlement first reaches a maximum about $15 \mathrm{~m}$ behind the tunnel face, and then, it decreases as the distance to the face increases. On the other hand, the axial horizontal displacement reaches a maximum about $25 \mathrm{~m}$ behind the tunnel face and vanishes for $Y=0$. These results are directly related to the existence of the boundaries of the model and do not correspond to any physical reality. Without a stabilization of the soil movement behind the tunnel face, it is very difficult to assess the final value of these displacements at a large distance behind the tunnel face. One possible solution would be to increase the model length in order to reduce the boundaries impact. This, however, will largely increase the computation time, by increasing both the number of excavation stages and the individual computation time of each stage. Probably this may be acceptable for purely deterministic analyses, but the present model is intended to be used in a probabilistic study which may involve a large number of computations of the deterministic model.

An alternative solution is proposed. It is based on the curves of Fig. 4, which depict the instantaneous settlement at the 35th excavation stage. This instantaneous settlement is computed as the difference between the total settlement at stage 35 and the one at stage 34 . It therefore represents

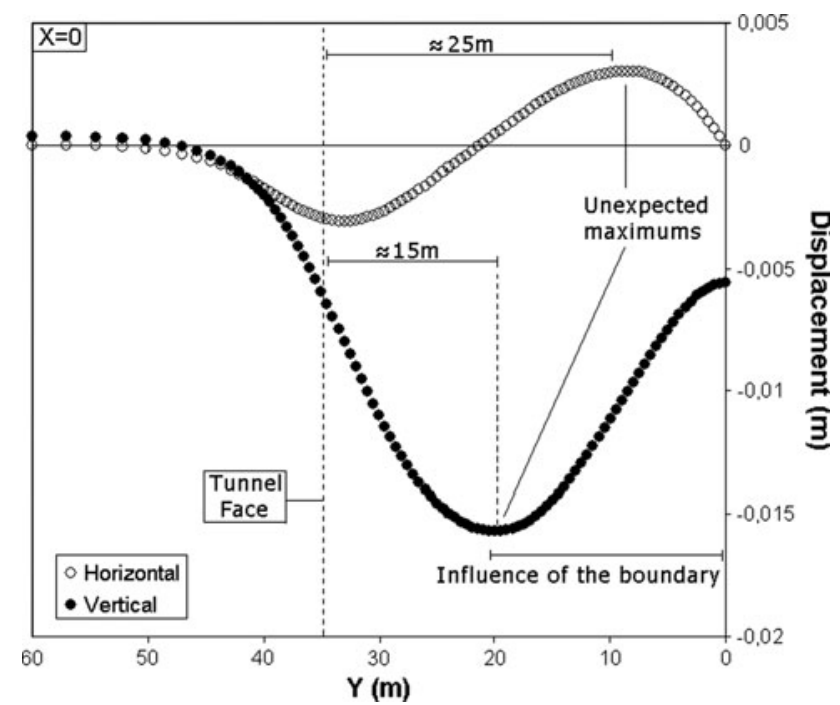

Fig. 3 Vertical settlements and horizontal displacements at the ground surface as provided directly by the numerical model 
the increment of settlement brought by the 35th stage of excavation. This figure (especially the settlement curve at the tunnel crown) provides some interesting information on the phenomena occurring during excavation. About $10 \mathrm{~m}$ in the upstream of the tunnel face, a progressive decompression of the soil is observed due to the face excavation, although this decompression is limited by the applied face pressure. This decompression triggers a beginning of settlement. In the neighborhood of the cutting wheel, a much larger settlement appears because of the overcutting. Behind this "peak", a small increase in settlement related to the conicity of the shield appears. The grout injection pressure at the tail void induces a negative settlement (i.e., a soil heave) at the end of the shield, and the grout consolidation and solidification induces a last settlement peak. It should be noticed here that if a more complex constitutive model with a different value of the stiffness was to be used in the unloading path, the effects of the grout pressure and the grout solidification may be much less important. For the curves corresponding to smaller depths, one may observe a progressive smoothing and attenuation of the settlement curves, until a rather regular settlement curve is obtained at the ground surface. This curve runs from $15 \mathrm{~m}$ in the upstream of the tunnel face to $25 \mathrm{~m}$ in the downstream of this face. Figure 4 also shows that the instantaneous settlement curves at the 35th stage are not disturbed by the model boundaries, the settlements being equal to zero at $Y=0 \mathrm{~m}$ and $Y=60 \mathrm{~m}$, for all depths. The total length of influence of a single excavation stage is thus smaller than $60 \mathrm{~m}$. It may therefore be considered that the tunnel has reached some kind of permanent state of excavation and that a new excavation stage would produce the same instantaneous settlement curves but $1 \mathrm{~m}$ further. This property is the reason why a number of 35 excavation steps were chosen and may be used to assess the final settlement directly by simple summation of the instantaneous settlement curves. Indeed, the settlement at any coordinate $Y$ is equal to the sum of the previous instantaneous settlements induced at this point by all the stages that were performed. This method can be generalized to the computation of the displacement of any point of the model. It should be emphasized that the summation method presented here does not rely on any assumption regarding to the elastic or plastic nature of the ground deformation. Indeed, it does not make use of any additive property of the elastic deformation. Its only assumption is that the excavation step chosen for the summation fully represents some kind of "permanent regime" of excavation. Under such an assumption, there is no reason for an excavation step to be different from another, and their induced displacements can therefore be summed.

Figure 5 depicts the vertical displacement and the horizontal displacement (in the $Y$ direction) of the points located at the ground surface along the $Y$ axis (a) as provided directly by the $\mathrm{FLAC}^{\mathrm{BD}}$ model and (b) as obtained by integration of the instantaneous displacements. It clearly appears that the method of integration of the instantaneous displacements allows one to accurately assess the final values of these displacements despite the presence of the model boundaries. The only shortcoming of this method is that it would not be adequate in the case of spatially varying soil properties, because the instantaneous displacements would not be the same from one stage to the other. This is not the case in the present study which only focuses on homogeneous soils, and the summation method is therefore perfectly relevant.

Figure 6 presents the settlement curves along the $Y$ axis, for several depths running from the ground surface to the tunnel crown, as obtained by using the method of integration. At a depth of $10 \mathrm{~m}$ (i.e., at the crown), the mechanisms induced by the excavation clearly appear, as was the case in Fig. 4: (a) a decompression of the soil in the upstream of the tunnel face, (b) a large settlement in the excavation chamber due to overcutting, (c) a linear increase of the settlement along the shield, (d) a local reduction of the settlement behind the shield (related to the grout injection pressure), and finally (e) an increase of the settlement (related to the solidification and consolidation of the grout), before a stabilization of the settlement about $20 \mathrm{~m}$ behind the face. Notice that the perfect linearity in the increase of the settlement along the shield is related to a contact between the soil and the external surface of the shield, which only occurs in the central part because of the triangular pressures applied by the migrating fluids at the front and at the rear of the shield. Notice also that the curve of settlement is attenuated and smoothed when the depth decreases and that the settlement curve at the surface is much more regular than the one at the tunnel crown. The final ground settlement after excavation is equal to $19.9 \mathrm{~mm}$.

Figure 7 provides two three-dimensional settlement curves as given by the method of integration. More precisely, Fig. 7 a depicts the instantaneous settlement curve at the 35th stage of excavation, and Fig. 7b presents the final settlement curve (with a vertical scale contracted 10 times with respect to Fig. 7a). Figure $7 \mathrm{~b}$ shows that the 3D settlement is the juxtaposition of a longitudinal (along $Y$ ) and of a transversal (along $X$ ) troughs. It appears that the complexity of the soil movements cannot be handled by only considering the transversal trough and that a 3D simulation of the excavation is more relevant than the classical plane strain 2D simulations ([1, 7, 13]).

Finally, it should be mentioned that the average computation time of the deterministic model is close to 150 min when using a $2.4 \mathrm{GHz}$ Quad-core CPU.

\subsection{Other soil movements}

When dealing with the impact of a tunnel excavation, one should not only consider the maximal settlement. 


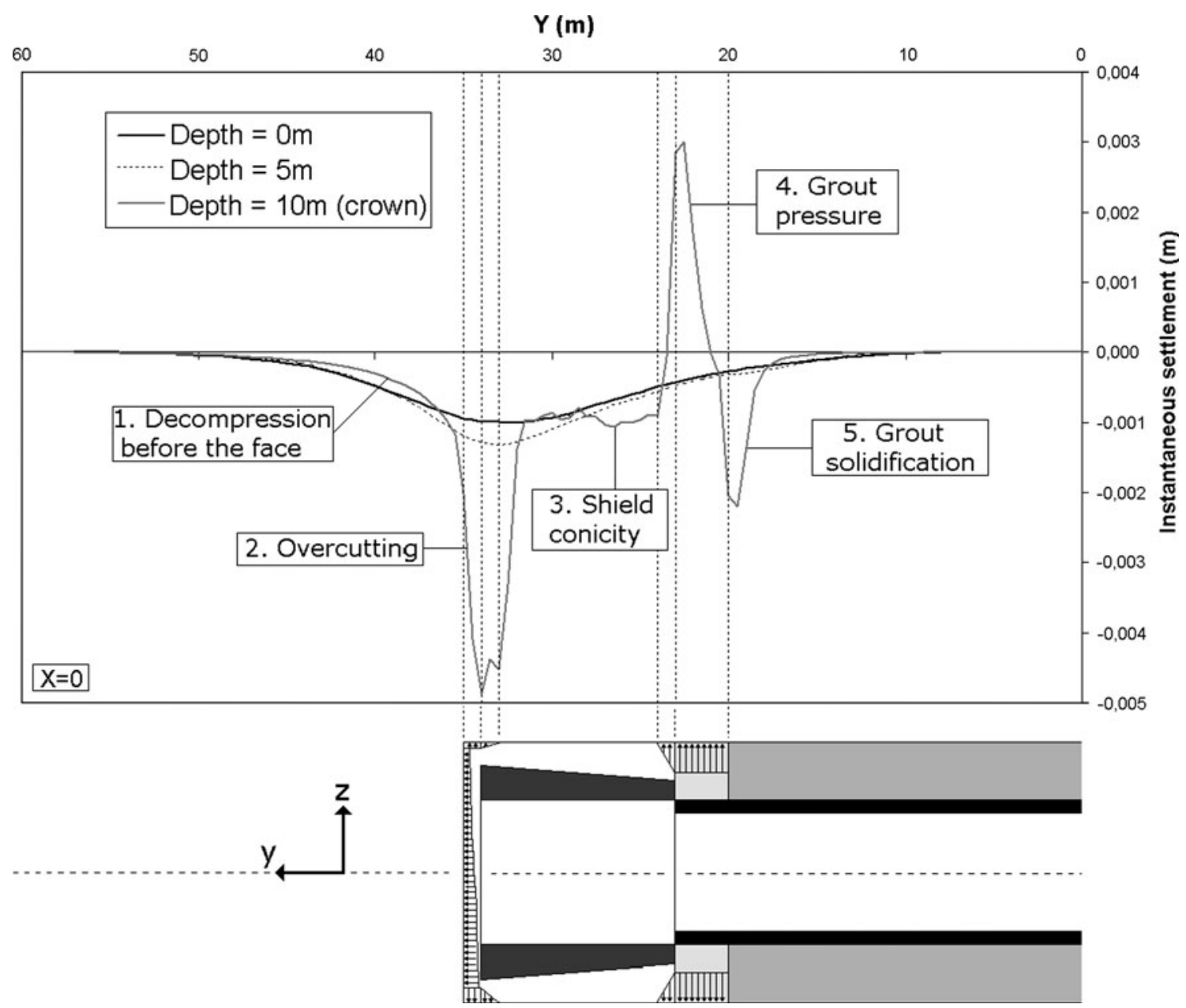

Fig. 4 Instantaneous settlements induced along the tunnel axis by the 35 th excavation stage

Indeed, other quantities should be taken into account, such as the slopes of the settlement curves (in longitudinal and transversal directions), the settlement distortions (in longitudinal and transversal directions), the horizontal displacements (along $X, Y$, or any other horizontal axis), or the horizontal deformations of the soil (in compression and extension). Overall, 12 output variables (called $S_{1}$ to $S_{12}$ ) are chosen to describe the ground movements around the excavation. These variables are listed in the first column of Table 1.

To illustrate the 12 output variables $\left(S_{1}\right.$ to $\left.S_{12}\right)$, Fig. 8 provides some curves obtained from the deterministic model by the summation method presented earlier. Figure $8 \mathrm{a}$ presents the displacements at the ground surface in the $X, Y$, and $Z$ directions in both the plane of symmetry of the tunnel (i.e., for $X=0$ ) and in a crosssection far beyond the tunnel face (i.e., for $Y=0$ ). The variables $S_{1}$ (maximal settlement), $S_{3}$ (maximal longitudinal displacement), and $S_{4}$ (maximal transversal displacement) are reported in Fig. 8a. The variable $S_{2}$ (maximal horizontal displacement) does not appear in this figure because this displacement is a combination of two displacements along $X$ and $Y$ and occurs outside of the planes $X=0$ and $Y=0$. Notice that, in the plane $X=0$, the displacements along $X$ are null because of the prescribed boundary conditions of the model due to symmetry.

Figure $8 \mathrm{~b}$ presents (a) the slopes and (b) the horizontal deformations at the ground surface. These curves were obtained by spatial derivation of the displacements provided by the summation method. Variables $S_{5}$ and $S_{6}$ correspond to the maximal slopes of the settlement trough in the longitudinal and transversal directions. The positions of the point where these maximal slopes occur are called $S_{7}$ and $S_{8}$. Namely, $S_{7}$ is the position of the point of maximal longitudinal slope with respect to the tunnel face $\left(S_{7}\right.$ may therefore be negative), while $S_{8}$ is the distance between the tunnel plane of symmetry and the point of maximal 


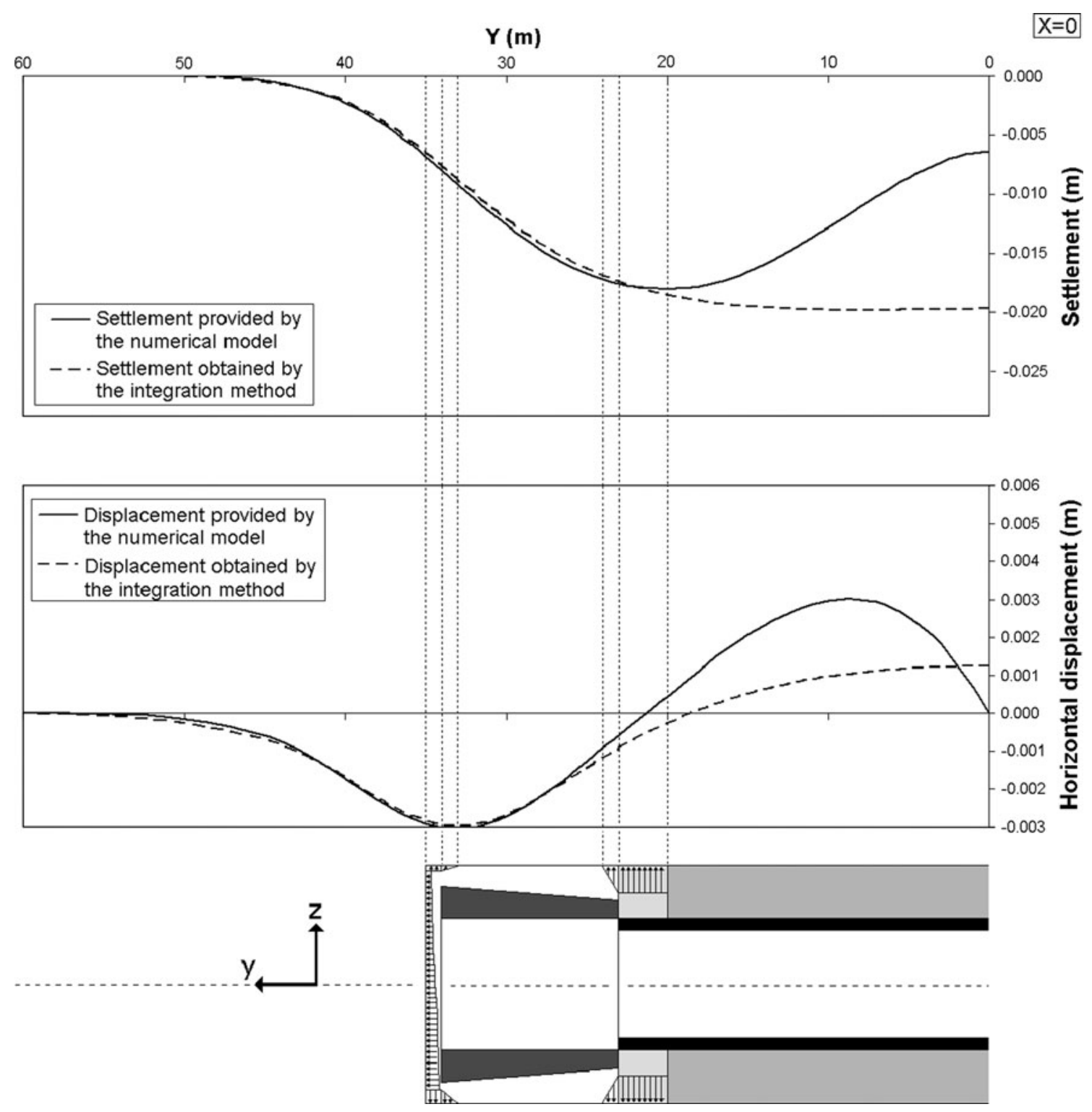

Fig. 5 Comparison between the settlements and horizontal displacements as provided directly by the numerical model and by the summation method

transversal slope. Finally, the variables $S_{9}$ to $S_{12}$ define the extreme values of the horizontal deformations at the ground surface in extension and in compression in both the transversal and the longitudinal directions.

To summarize, the variables $S_{1}$ to $S_{4}$ are related to the maximum displacements, the variables $S_{5}$ to $S_{8}$ are related to the geometry of the settlement trough, and the variables $S_{9}$ to $S_{12}$ are related to the extreme horizontal deformations of the soil at the ground surface. The values of these 12 parameters are computed and stored in the second column of Table 1 for the reference case studied in this section (i.e., $c=7 \mathrm{kPa}, \varphi=17^{\circ}, \psi=0^{\circ}, \gamma=18 \mathrm{kN} / \mathrm{m}^{3}$, $E=50 \mathrm{MPa}, v=0.3, E_{\text {grout }}=10 \mathrm{MPa}, \sigma_{t}=190 \mathrm{kPa}$, $\sigma_{i n j}=215 \mathrm{kPa}$, and $L_{i n j}=3 \mathrm{~m}$ ).

\section{Overview on the conventional and the extended CSRSM and Sobol indices for the probabilistic analyses}

As is well known, the most robust probabilistic approach is the Monte-Carlo simulation method. This method makes possible the propagation of the uncertainties from the input data to the system output through a deterministic model to compute the PDF of the system response or the system failure probability. Notice, however, that this method requires a large number of calls of the deterministic model (e.g., about 1,000,000 samples for a target failure probability of $10^{-5}$ ). This number of calls is obviously out of reach for the deterministic numerical model described in a 


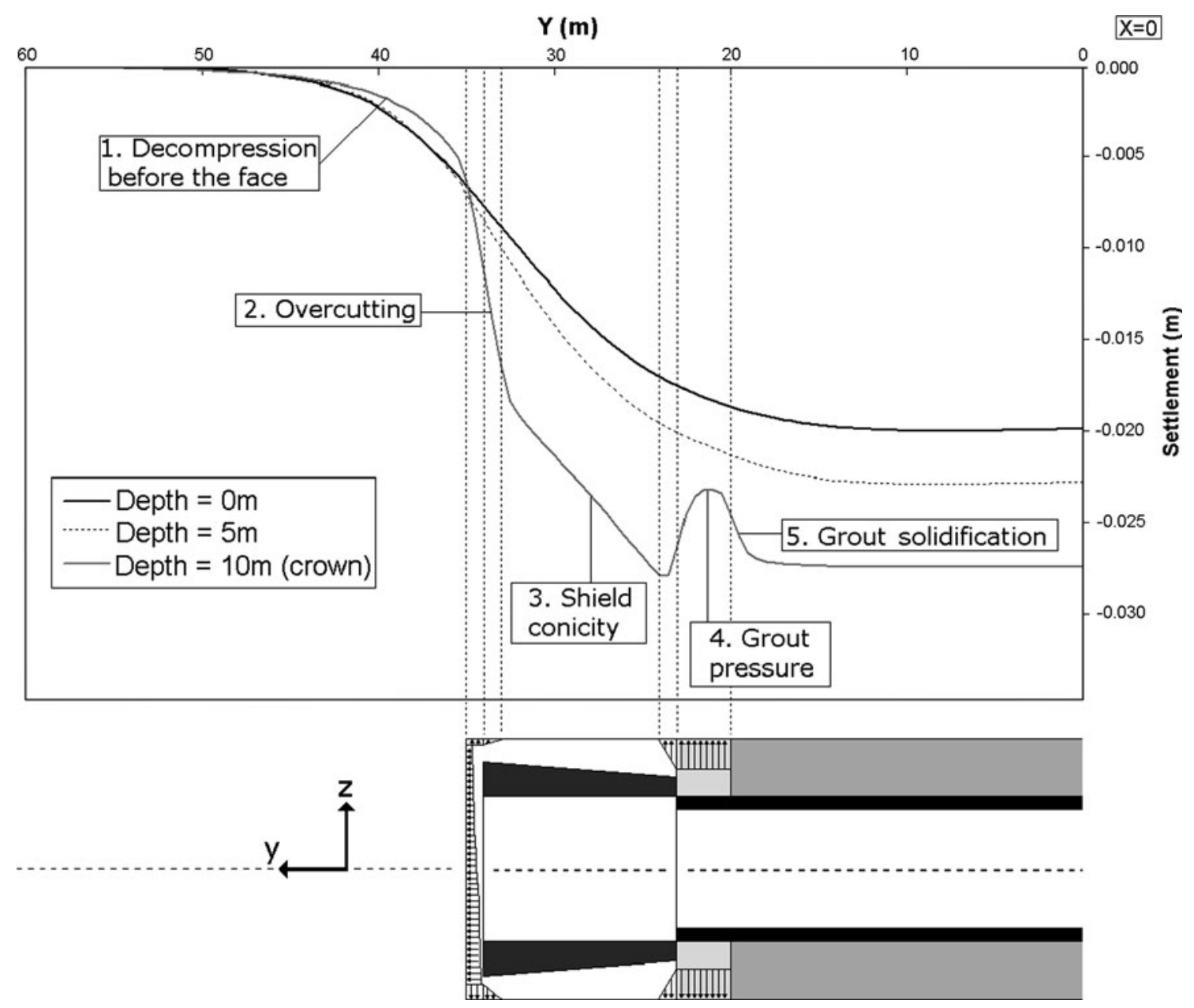

Fig. 6 Total settlements along the tunnel axis after the 35th excavation stage

previous section. To overcome this shortcoming, a powerful probabilistic method called Collocation-Based Stochastic Response Surface Methodology (CSRSM) is used in this paper. This method is briefly described herein. The reader may find a more detailed description in the references [8-10, 16, 17, 24, 25, 28, 31, 32].

The CSRSM allows one to obtain an analytical approximation (called meta-model) of a random system response. Thus, the system response may be computed more quickly in any classical probabilistic method (such as Monte-Carlo Sampling) to characterize the variability of this response, because one uses the meta-model and not the original deterministic model. In the framework of this method, the meta-model is a Polynomial Chaos Expansion (PCE) of a given order $\mathrm{n}$. The meta-model is expressed in this paper in the basis of the multidimensional Hermite polynomials of orders $\leq n$, although other types of polynomials may also be used (cf. [34]). For a given set of the probabilistic parameters of the input random variables, the unknown coefficients of the PCE are obtained in this paper by regression using the response of the original deterministic model at a given number of so-called collocation points. It should be mentioned that when the number of random variables is important, the number of the available collocation points becomes much larger than the number of the unknown coefficients. It is therefore necessary to reduce the number of collocation points to be used in the regression process, in order to keep a good accuracy of the meta-model without increasing the computation time. Several methods exist $([10,32])$ to determine the most suitable collocation points (number and positions) to be used in the computations.

It should be noted here that the conventional CSRSM described above is an efficient and accurate tool for the probabilistic study of a mechanical model, but it suffers from the fact that its formalism implies a new set of deterministic computations each time one of the probabilistic parameters of the input variables (such as the COV, the correlation coefficient or the type of the probability distribution) is changed. This is because the position of the collocation points in the physical space is dependant on the correlation matrix and the CDF of the input variables. 


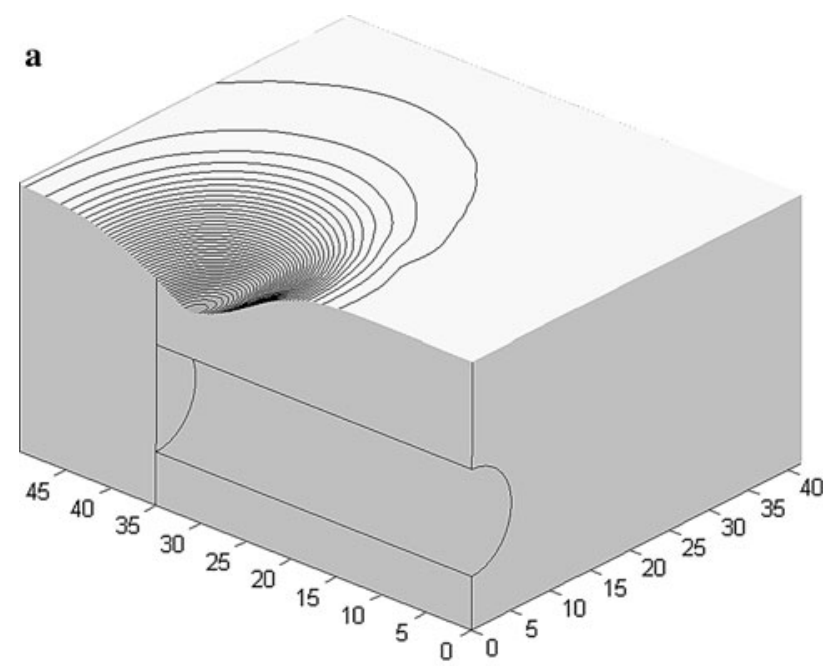

The time cost of a parametric study (when using a computationally expensive model like the one proposed in the present paper) will therefore be very high, while such a study may be necessary because these parameters are usually not known with a great accuracy. An extension of CSRSM called E-CSRSM was proposed in [25] and will be used in this paper. This method makes possible the use of a unique set of collocation points and thus a unique set of deterministic computations to carry out a parametric study. This significantly reduces the computation time of the parametric study. The E-CSRSM is based on the fact that the position of the collocation points (determined using the roots of the Hermite polynomials) is not mandatory. For more details on this method, the reader may refer to [25].

Once a PCE was determined, a global sensitivity anal-

b

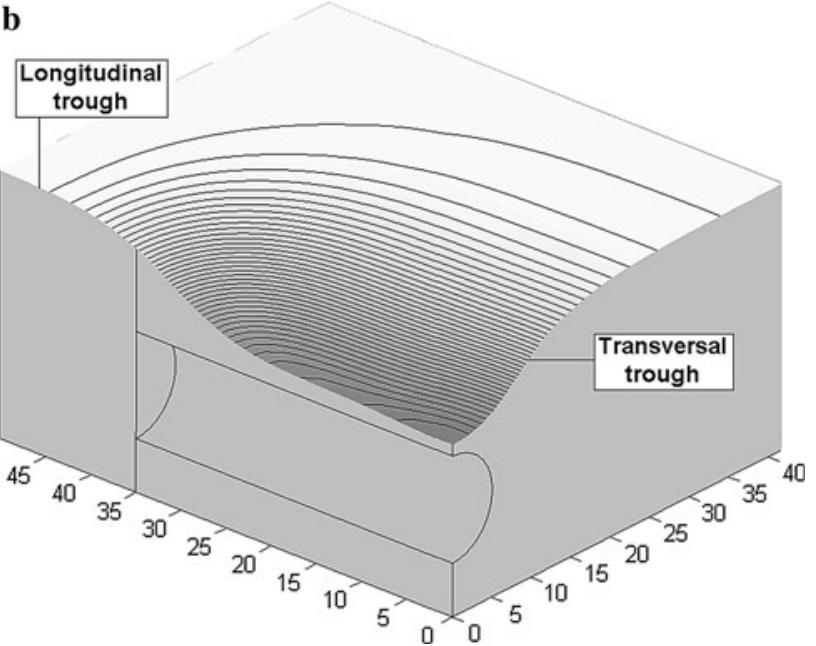
ysis of the system response can be performed analytically by computing the Sobol index of each random variable ([32]) using the coefficients of this PCE. The Sobol index of a random variable provides the contribution of this variable to the total variance of the response. Consequently, the sum of all the Sobol indices of a given system response is equal to 1 . Details on the computation of the Sobol indices may be found in [24].

\section{Probabilistic analysis of the ground movements induced by excavation}

Because of the complexity of the phenomena occurring during a pressurized excavation, the numerical model uses a large number of input variables, which can exhibit a wide range in their variability. In order to take into account the randomness of the uncertain parameters in the probabilistic analysis, seven input variables were considered as independent random variables and the other input variables

Table 1 Deterministic and probabilistic mean values of the 12 output variables

\begin{tabular}{llll}
\hline Output variable & Symbol of the output variable & Deterministic mean value & Probabilistic mean value \\
\hline Maximal settlement $(\mathrm{mm})$ & $S_{1}$ & 19.90 & 20.9 \\
Maximal horizontal displacement $(\mathrm{mm})$ & $S_{2}$ & 8.53 & 9.09 \\
Maximal longitudinal displacement $(\mathrm{mm})$ & $S_{3}$ & 1.81 & 1.99 \\
Maximal transversal displacement $(\mathrm{mm})$ & $S_{4}$ & 8.43 & 9.00 \\
Maximal longitudinal slope $(\mathrm{mm} / \mathrm{m})$ & $S_{5}$ & 1.22 & 1.23 \\
Maximal transversal slope $(\mathrm{mm} / \mathrm{m})$ & $S_{6}$ & 1.50 & 1.57 \\
Longitudinal $i$ parameter $(\mathrm{m})$ & $S_{7}$ & -2.50 & -2.46 \\
Transversal $i$ parameter $(\mathrm{m})$ & $S_{8}$ & 7.23 & 7.63 \\
Maximal longitudinal extension $(\mu \mathrm{m} / \mathrm{m})$ & $S_{9}$ & 337 & 339 \\
Maximal longitudinal compression $(\mu \mathrm{m} / \mathrm{m})$ & $S_{10}$ & -380 & -369 \\
Maximal transversal extension $(\mu \mathrm{m} / \mathrm{m})$ & $S_{11}$ & 420 & 462 \\
Maximal transversal compression $(\mu \mathrm{m} / \mathrm{m})$ & $S_{12}$ & $-1,754$ & $-1,773$ \\
\hline
\end{tabular}



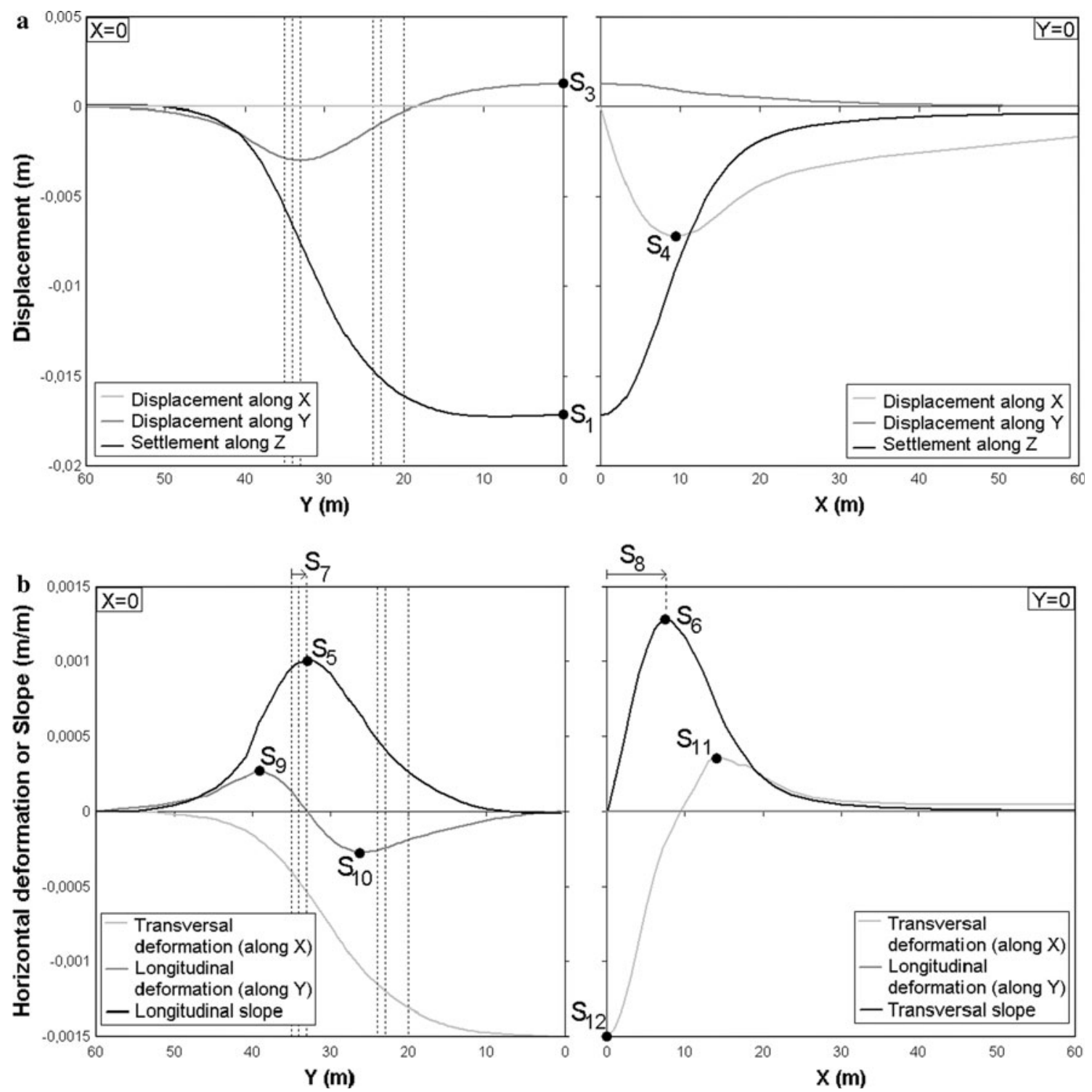

Fig. 8 Detail of the 12 output variables: a settlements and horizontal displacements in the planes $X=0$ and $Y=0$, b slopes and horizontal deformations in the planes $X=0$ and $Y=0$

were considered as deterministic. The deterministic input variables of the numerical model are related to the geometry of the excavation and the unit weight of the soil. On the other hand, the seven random variables considered in the analysis are provided in Table 2 with their illustrative mean values used in this paper. Three probabilistic scenarios (optimistic, neutral, and pessimistic) are considered since the variability of the input random variables is generally not known with accuracy. These scenarios are used to account for the amount of information that is available to the designer. The scenario is thus an indicator of the socalled epistemic error (related to a lack of knowledge), rather than of the natural variability of the soil. In a practical context, an optimistic scenario should be used if the tunnel is excavated in a well-known soil (such as the one in a city in which designers have a lot of experience from past projects), while a pessimistic scenario is more suitable for a project in which there is only a very small amount of data available on the soil properties. Finally, a neutral scenario is one for which the amount of information can be qualified as average or slightly above average. It should be emphasized here that the scenarios are closely linked not only to the quantity but also to the quality of the soil investigations, although the choice of a proper probabilistic scenario is a broad topic which is beyond the framework of the present study. All the values of the COVs for the three scenarios are provided in Table 2.

It should be mentioned that the variability of the soil parameters $(\varphi, c, E)$ is quite well documented ([29]). It is related to the natural variability of the soil and to the 
Table 2 Mean values, COVs, and types of distributions of the seven random input variables

\begin{tabular}{|c|c|c|c|c|c|}
\hline \multirow[t]{2}{*}{ Variable } & \multirow[t]{2}{*}{ Mean } & \multicolumn{3}{|l|}{$\operatorname{COV}(\%)$} & \multirow[t]{2}{*}{ Type of distribution } \\
\hline & & Optimistic scenario & Neutral scenario & Pessimistic scenario & \\
\hline Internal friction angle $\varphi\left({ }^{\circ}\right)$ & 17 & 5 & 10 & 15 & Gaussian or Beta \\
\hline Cohesion $c(\mathrm{kPa})$ & 7 & 10 & 20 & 30 & Gaussian or Lognormal \\
\hline Young modulus of the soil $E(\mathrm{MPa})$ & 50 & 10 & 15 & 20 & Gaussian or Lognormal \\
\hline Young modulus of the grout $E_{\text {grout }}(\mathrm{MPa})$ & 10 & 30 & 40 & 50 & Gaussian or Lognormal \\
\hline Applied face pressure $\sigma_{t}(\mathrm{kPa})$ & 190 & 5 & 15 & 25 & Gaussian or Lognormal \\
\hline Grout pressure $\sigma_{i n j}(\mathrm{kPa})$ & 215 & 5 & 15 & 25 & Gaussian or Lognormal \\
\hline Grout solidification length $L_{i n j}(\mathrm{~m})$ & 3 & 30 & 40 & 50 & Gaussian or Lognormal \\
\hline
\end{tabular}

possible measurement errors. The COV values of these parameters are chosen according to the results of [29], for the three scenarios. The uncertainty on the elastic modulus of the injected grout $E_{\text {grout }}$ is much more important since this parameter is used to model several complex phenomena which are not perfectly understood: grout consolidation, fluid migration in the soil around the lining, progressive hardening of the grout, etc. The large values of $\mathrm{COV}$ used for $E_{\text {grout }}$ are therefore related to a large model uncertainty. The COV of the fluid pressures $\left(\sigma_{t}\right)$ and $\left(\sigma_{i n j}\right)$ applied, respectively, at the tunnel face and behind the shield are very low (5\%) in the optimistic scenario, and quite high $(25 \%)$ in the pessimistic one, depending on the level of control of these pressures (related to the tunnel machinery and human calibration). This variability also accounts for the uncertainty of the soil behind these pressures, especially its permeability to the retaining fluid. The COVs used for the solidification length $L_{i n j}$ are very high because this length is related to the time of solidification of the grout and the advance rate of the tunnel boring machine, which are both very uncertain.

In the present paper, each input random variable will be modeled using two different statistical distributions. In the so-called case of "normal variables", the seven variables are considered as Gaussian, while in the so-called case of "non-normal variables", they are considered as following bounded distributions. In that case, the soil friction angle $\varphi$ is assumed to follow a beta distribution bounded by $8^{\circ}$ and $35^{\circ}$, and the six other variables are assumed to follow a lognormal distribution (which is non-negative by nature, and therefore very convenient and very commonly used for the modeling of physical variables which cannot be smaller than zero).

For a given realization of the input random variables, the numerical model provides all the soil movements around the excavation. Among the large amount of data, 12 system responses $\left(S_{1}, S_{2}, \ldots, S_{12}\right)$ were monitored as explained in a previous section. The output variables $S_{1}$ to $S_{4}$ are related to the extreme displacements at the ground surface in various directions, the variables $S_{5}$ and $S_{6}$ are related to the maximum slopes at the ground surface, the variables $S_{7}$ and $S_{8}$ are related to the dimensions of the settlement trough, and the variables $S_{9}$ to $S_{12}$ are related to the extreme values of the horizontal deformations at the ground surface.

The CSRSM is used in its conventional formalism to determine the coefficients of the PCE in the case of normal input variables and a neutral probabilistic scenario. For two PCEs of order 3 and 4 (with 7 random variables for both orders), the number of unknown coefficients and the number of the available collocation points are given in Table 3. The number of unknown coefficients for the PCE of order 4 appears out of reach with conventional computational means, and thus, the order 3 is chosen for the present study. This choice does not reduce the accuracy of the PCE approximation in the central part of the distributions (i.e., around the mean values of the output variables), but it may make it a bit less accurate at the distribution tails (i.e., when the values taken by the output variables are quite far from their mean values). Notice finally that [24] have shown that a PCE of order 3 was able to accurately deal with failure probabilities of the order of magnitude of $10^{-4}$ with a good accuracy. This order is used in this paper. For smaller failure probabilities (about $10^{-5}$ ), a PCE of order 4 may be necessary to lead to sufficiently accurate results.

As it may be seen from Table 3, 16,384 collocation points are available for a PCE of order 3. A rational methodology based on the invertibility of the information matrix was proposed for the determination of the minimal necessary number of collocation points to be used in the

Table 3 Number of coefficients and of available collocation points for a seven variables PCE

\begin{tabular}{lcc}
\hline & PCE of order 3 & PCE of order 4 \\
\hline $\begin{array}{l}\text { Number of coefficients } \\
\begin{array}{l}\text { Number of available } \\
\text { collocation points }\end{array}\end{array}$ & 120 & 330 \\
\hline
\end{tabular}


computation (see [32]). According to this method, the collocation points defined in the standard space by a vector $\left(\xi_{1}, \xi_{2}, \xi_{3}, \xi_{4}, \xi_{5}, \xi_{6}, \xi_{7}\right)$ should be sorted by increasing order with respect to their distance $d$ to the mean point (origin of the standard space) where $d$ is given by:

$d=\sqrt{\xi_{1}^{2}+\xi_{2}^{2}+\xi_{3}^{2}+\xi_{4}^{2}+\xi_{5}^{2}+\xi_{6}^{2}+\xi_{7}^{2}}$

The collocation points retained by this method that lay the furthest from the mean point were at a distance $d_{\max }=2.96$ for a PCE of order 3 with 7 variables. It was chosen in this study to use all the available collocation points corresponding to $d \leq d_{\max }$ in order to respect the symmetry of the sampling scheme in the standard space. Thus, only 1,025 collocation points were used instead of the total number of 16,384. As mentioned before, the case of normal variables with a neutral scenario is firstly considered. The total computation time was about 107 days on a $2.4 \mathrm{GHz}$ Quad-core CPU. For more efficiency, these computations were launched simultaneously on several computers. Once these computations were finished, the 1,025 collocation points provided 12 PCEs for the 12 output variables. Remember that each PCE requires the determination of 120 unknown coefficients. These coefficients are only valid for the case of a neutral probabilistic scenario with normal variables. However, the use of E-CSRSM has allowed to obtain directly the PCE coefficients for the other scenarios (optimistic and pessimistic), and for the case of non-normal variables. Thus, for each one of the 12 output variables of the model, we have obtained six PCEs expressed in the standard space, with 120 coefficients for each one of these PCEs.

\section{Probabilistic results}

\subsection{Coefficients of variation}

For each one of the 12 output random variables, columns 3 and 4 of Table 1 provide, respectively, the deterministic values (using the mean values of the input variables) and the probabilistic mean values as obtained using a neutral probabilistic scenario and normal input variables. For all the variables, the deterministic and probabilistic approaches provide close values, which means that the randomness of the input variables leads to a variability of the output variables which is roughly centered on their deterministic values. This variability is provided in Fig. 9a in a dimensionless form (using the coefficient of variation defined as the ratio between the standard deviation $\sigma$ and the mean value $\mu$ ) for the 12 output variables and for the three scenarios when the input variables are considered as normally distributed. These COVs were computed using
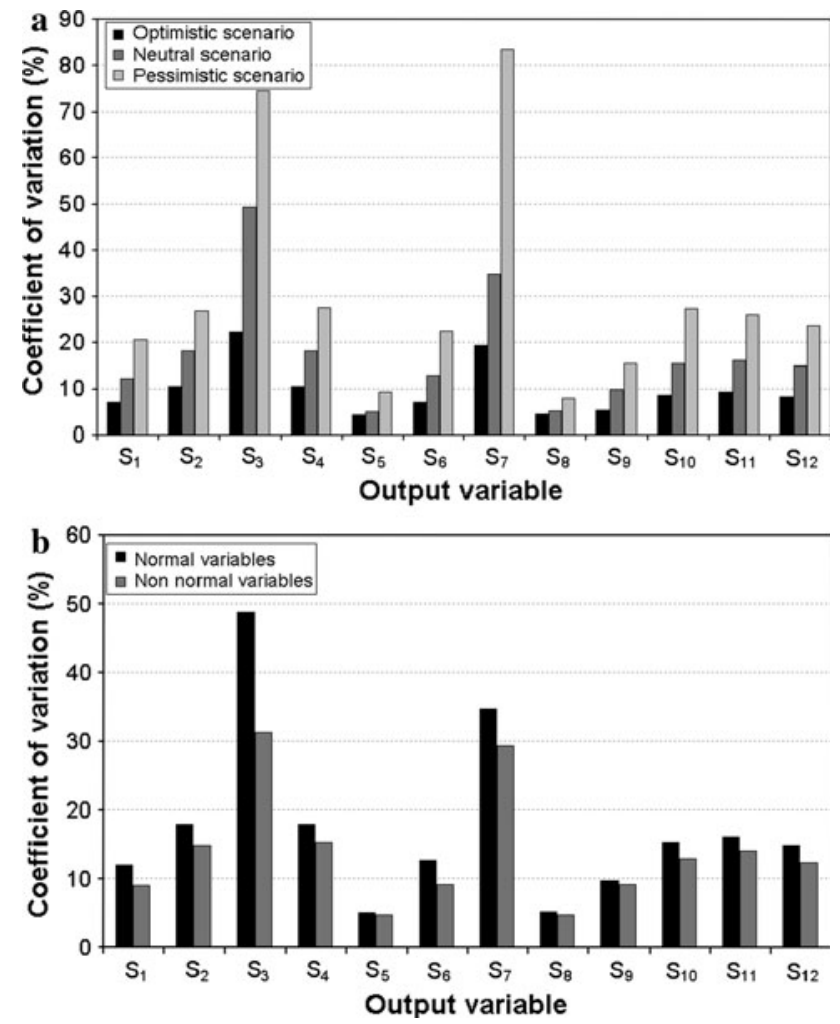

Fig. 9 Coefficients of variation of the 12 output variables: a influence of the probabilistic scenario, $\mathbf{b}$ influence of the type of distribution of the input variables

the first two statistical moments of the PDF obtained by Monte-Carlo samplings on the meta-model. As expected, the pessimistic scenario leads to the largest variability.

According to Fig. 9a, the maximal longitudinal displacement (variable $S_{3}$ ) and the parameter $i$ of the longitudinal settlement trough (variables $S_{7}$ ) are the most affected by the variability of the input variables, with coefficients of variation of 49 and $35 \%$, respectively, in the case of a neutral scenario. The maximum settlement (variable $S_{1}$ ) exhibits a moderate variability, with COVs of $7.0,12.1$, and $20.5 \%$ in the case of optimistic, neutral, and pessimistic scenarios, respectively. The longitudinal maximal slope (variable $S_{5}$ ) and the parameter $i$ of the transversal trough (variable $S_{8}$ ) are the variables with the smallest variability (5.2 and $5.6 \%$, respectively, in the neutral scenario). The remaining variables (i.e., the variables $S_{2}, S_{4}$, and $S_{6}$, and the ones corresponding to horizontal deformations) have similar dispersions with COV comprised between 10 and $16 \%$ in the case of a neutral scenario.

Figure $9 \mathrm{~b}$ presents the same kind of results for normal and non-normal variables in the case of a neutral probabilistic scenario. For all of the 12 output variables, it appears that the assumption of normal variables leads to a 


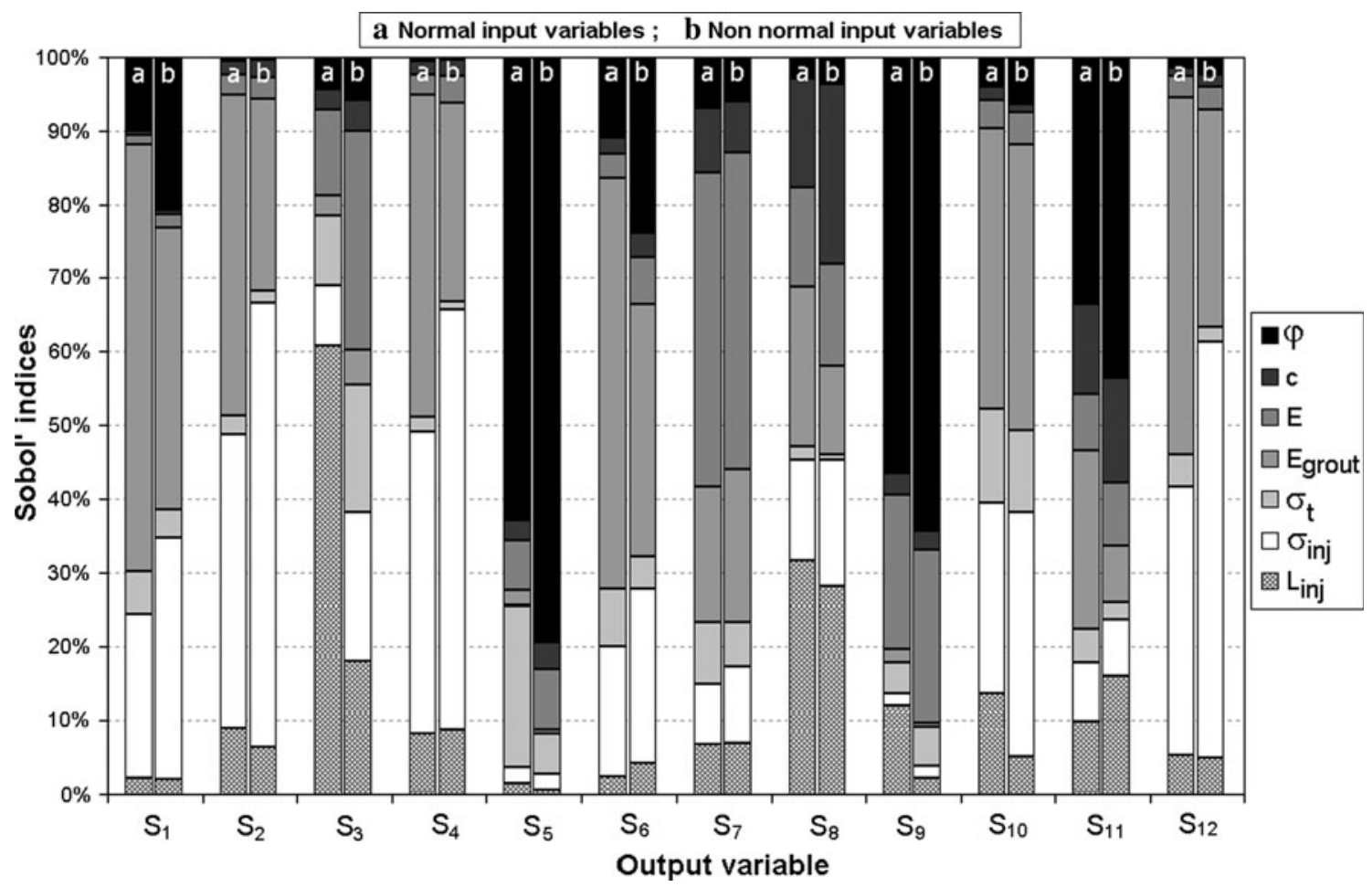

Fig. 10 Sobol indices of the 12 output variables with respect to the 7 input variables, for a neutral probabilistic scenario

larger dispersion. The assumption of a Gaussian distribution of the input variables is therefore conservative.

\subsection{Sobol indices}

Figure 10 presents the values of Sobol indices computed for normal and non-normal input variables in the neutral scenario. For each one of the 12 output variables, the Sobol indices of the seven random variables are presented in a cumulated way from 0 to $100 \%$.

Figure 10 clearly shows that for some system responses, the Sobol indices may be very different depending on the statistical distribution of the input variables. For example, the value of $S U_{1}(\varphi)$ (i.e., Sobol index of $\varphi$ for the variable $S_{1}$, corresponding to the maximal settlement) is twice larger if the input variables are non-normal than if they are normal, although the mean values and COVs of these input variables are equal in both cases. On the other hand, the Sobol indices of other system responses (for example $S_{7}$ and $S_{10}$ ) seem quite unaffected by the type of distribution of the input variables.

Besides, Fig. 10 shows that the variability of the maximal settlement (variable $S_{1}$ ) is mainly related to the one of the modulus of the grout $\left(S U_{1}\left(E_{\text {grout }}\right)=61.7 \%\right.$ for normal variables) and that of the grouting pressure $\left(S U_{1}\left(\sigma_{i n j}\right)=23.8 \%\right.$ for normal variables $)$. This means that most of the uncertainty on the maximal settlement may be removed by accurately controlling and modeling the phenomena occurring at the tail void of the shield (i.e., the grout pressure and consolidation). Figure 10 also shows that the uncertainty on the maximal horizontal displacement (variable $S_{2}$ ) is mostly related to the same variables as the maximal settlement, that is, $E_{\text {grout }}$ and $\sigma_{i n j}$. The variability of the maximal longitudinal slope (variable $S_{5}$ ) and that of the extreme horizontal deformations in extension (variables $S_{9}$ and $S_{11}$ ) are mostly related to the variability of the soil parameters ( $\varphi$ and $E$ ), while the variability of the maximal transversal slope (variable $S_{6}$ ) and the extreme horizontal deformations in compression (variables $S_{10}$ and $S_{12}$ ) are much more related to the uncertainty of the tail void phenomena $\left(E_{\text {grout }}\right.$ and $\left.\sigma_{i n j}\right)$. Finally, the variability of the parameters $i$ (variables $S_{7}$ and $S_{8}$ ) that describe the shape of the settlement trough in the longitudinal and transversal directions, respectively, arises from the variability of all the input variables, since all the Sobol indices have a significant value. This shows that the 3D trough geometry is related to a number of complex phenomena involving all the input variables. To summarize, Fig. 10 emphasizes the fact that none of the input variables may be neglected if one wants to assess the variability of all the output variables of the system. However, when focusing on the variability of a given output variable, Fig. 10 shows that it is possible to use only a limited number of random variables, since the variability of the others has no impact on the variability of the output variable. For example, it appears that the variability of the maximal ground 
settlement $\left(S_{1}\right)$ may be assessed using only $\varphi, E_{\text {grout }}, \sigma_{t}$, and $\sigma_{i n j}$ as random variables, since the cumulated value of the Sobol indices of the three other input variables $(c, E$, and $L_{i n j}$ ) remains smaller than $5 \%$ both for normal and nonnormal variables. These three variables may thus be considered as deterministic. Figure 10 may therefore be used as a tool to reduce the number of random variables for a given problem.

The small value of the Sobol index of $E$ may appear striking, because the elastic modulus is known to have a large influence on the tunneling-induced ground settlement. However, this low value does not mean that $E$ does not have an impact on the settlement, but only means that a very little part of the variability of the settlement is related to the variability of $E$. In other words, despite the well-known deterministic influence of $E$ on the settlement, the variability of $E$ does not induce a large amount of variability of the settlement. This is related to the relatively low value of the commonly observed COV of $E$ (equal to $15 \%$ ) that was used in this probabilistic scenario.

\subsection{Cross-correlation}

This section aims at investigating the existence of possible correlations between the 12 output variables. The case of normal variables and of a neutral probabilistic scenario was considered in the analysis. Monte-Carlo simulation method using $10^{5}$ samples was used to perform the computations. The correlations are provided in the crosscorrelation matrix given in Table 4. It should be noted that an absolute value was adopted for the negative variables (variables $S_{7}, S_{10}$, and $S_{12}$ ) shown in Table 1 in order to obtain positive correlation coefficients which have more physical meaning. The correlation coefficient between two variables is useful to assess if two output variables have similar statistical behavior. For example, the maximal settlement (variable $S_{1}$ ) and the maximal horizontal displacement (variable $S_{2}$ ) have a very high correlation coefficient (0.95). This means that high values of these two output variables are likely to occur simultaneously and that the damage effects of these high values are likely to be cumulated.

Table 4 shows that the maximums ground displacements (variables $S_{1}$ to $S_{4}$ ) seem to be quite well correlated among each other, as well as the maximum slopes of the settlement trough (variables $S_{5}$ and $S_{6}$ ) and the trough widths (parameters i, variables $S_{7}$ and $S_{8}$ ). On the contrary, the extreme horizontal ground deformations are less correlated among each other.

It may also be observed from Table 4 that there are important correlations between variables $S_{1}, S_{6}$, and $S_{8}$ (i.e., maximum settlement, transversal slope, and transversal $i$ parameter), while the correlations are much smaller between variables $S_{1}, S_{5}$, and $S_{7}$ (i.e., maximum settlement, longitudinal slope and longitudinal $i$ parameter). One may deduce from this observation that the geometry (width and slope) of the longitudinal settlement trough is hardly related to the value of the maximal settlement, while the geometry of the transversal settlement trough is strongly related to this value.

\section{Probabilities of failure}

This section considers six different modes of failure. Following the usual formalism of the reliability theory, a failure mode is defined by a function of performance called G. This function depends (a) on the output value provided by the chosen deterministic model and (b) on the admissible threshold for this output. $G$ may have any shape and formulation, provided that it is strictly positive if the system is safe, and strictly negative if the system is at failure. Thus, for a given set of the input variables, the negative or positive sign of $G$ is a direct indicator of the failure or safety of the system. In the present study, each of the six possible failure modes is assigned a different performance function $G$. Thus, for a given failure mode and a given failure threshold, the probability of failure is equal to the probability to obtain $G<0$ after running the model (or the meta-model in the present case).

The first failure mode proposed in this study is called mode $\mathrm{A}$ and is related to the exceeding of a maximal admissible settlement $v_{\max }$. The corresponding function of performance is:

$G_{A}=v_{\max }-S_{1}$

The failure mode $B$ is defined by the exceeding of a maximal admissible horizontal displacement $\delta_{\max }$, with the following function of performance:

$G_{B}=\delta_{\max }-S_{2}$

The failure mode $C$ is defined by the exceeding of a maximal admissible slope $p_{\max }$ of the settlement trough, either in the transversal or in the longitudinal direction, with the following function of performance:

$G_{C}=p_{\max }-\max \left(S_{5}, S_{6}\right)$

The failure mode $D$ is defined by the exceeding of a maximal admissible distortion $\gamma_{\max }$ and is therefore related to the value of the maximum settlement and the dimensions of the settlement trough. Since the point of the settlement trough for which the settlement is null is difficult to estimate accurately, an arbitrary measure of the distortion is chosen here, using the distance between the point of maximal settlement and the point of maximum slope of the 
Table 4 Cross-correlation matrix of the 12 output random variables

\begin{tabular}{|c|c|c|c|c|c|c|c|c|c|c|c|c|}
\hline & \multicolumn{12}{|c|}{ Output variable } \\
\hline & $S_{1}$ & $S_{2}$ & $S_{3}$ & $S_{4}$ & $S_{5}$ & $S_{6}$ & $S_{7}$ & $S_{8}$ & $S_{9}$ & $S_{10}$ & $S_{11}$ & $S_{12}$ \\
\hline \multicolumn{13}{|c|}{ Output variable } \\
\hline$S_{1}$ & 1 & 0.95 & 0.43 & 0.96 & 0.54 & 0.99 & 0.50 & 0.76 & 0.37 & 0.56 & 0.90 & 0.97 \\
\hline$S_{2}$ & 0.95 & 1 & 0.52 & 1.00 & 0.40 & 0.93 & 0.42 & 0.61 & 0.18 & 0.41 & 0.79 & 0.98 \\
\hline$S_{3}$ & 0.43 & 0.52 & 1 & 0.50 & 0.25 & 0.43 & 0.08 & 0.12 & 0.46 & 0.49 & 0.35 & 0.46 \\
\hline$S_{4}$ & 0.96 & 1.00 & 0.50 & 1 & 0.39 & 0.93 & 0.41 & 0.62 & 0.19 & 0.41 & 0.79 & 0.99 \\
\hline$S_{5}$ & 0.54 & 0.40 & 0.25 & 0.39 & 1 & 0.57 & 0.54 & 0.40 & 0.53 & 0.54 & 0.65 & 0.47 \\
\hline$S_{6}$ & 0.99 & 0.93 & 0.43 & 0.93 & 0.57 & 1 & 0.49 & 0.77 & 0.36 & 0.62 & 0.94 & 0.94 \\
\hline$S_{7}$ & 0.50 & 0.42 & 0.08 & 0.41 & 0.54 & 0.49 & 1 & 0.61 & 0.42 & 0.32 & 0.41 & 0.50 \\
\hline$S_{8}$ & 0.76 & 0.61 & 0.12 & 0.62 & 0.40 & 0.77 & 0.61 & 1 & 0.44 & 0.61 & 0.71 & 0.66 \\
\hline$S_{9}$ & 0.37 & 0.18 & 0.46 & 0.19 & 0.53 & 0.36 & 0.42 & 0.44 & 1 & 0.15 & 0.45 & 0.26 \\
\hline$S_{10}$ & 0.56 & 0.41 & 0.49 & 0.41 & 0.54 & 0.62 & 0.32 & 0.61 & 0.15 & 1 & 0.65 & 0.41 \\
\hline$S_{11}$ & 0.90 & 0.79 & 0.35 & 0.79 & 0.65 & 0.94 & 0.41 & 0.71 & 0.45 & 0.65 & 1 & 0.82 \\
\hline$S_{12}$ & 0.97 & 0.98 & 0.46 & 0.99 & 0.47 & 0.94 & 0.50 & 0.66 & 0.26 & 0.41 & 0.82 & 1 \\
\hline
\end{tabular}

settlement trough (either in the transversal or longitudinal direction). In the transversal direction, this distance is equal to the parameter $i$ of the transversal trough. In the longitudinal direction, this distance is taken as the one between the point of maximal slope of the longitudinal settlement trough and an arbitrary point located $20 \mathrm{~m}$ behind the tunnel face. The observation of the settlement trough (Fig. 6) indeed shows that one may consider that the maximal settlement is reached at this point. If $S_{1}^{\prime}$ is the value of the settlement at the point of maximal slope of the longitudinal trough and $S_{1}^{\prime \prime}$ is the value of the settlement at the point of maximal slope of the transversal trough, the function of performance of this failure mode is:

$G_{D}=\gamma_{\max }-\max \left(\frac{S_{1}-S_{1}^{\prime}}{S_{7}+20 m}, \frac{S_{1}-S_{1}^{\prime \prime}}{S_{8}}\right)$

Moreover, the following simple assumption may be used:

$S_{1}^{\prime}=S_{1}^{\prime \prime}=S_{1} / 2$

Finally, the performance function is:

$G_{D}=\gamma_{\max }-\max \left(\frac{0.5 \cdot S_{1}}{S_{7}+20 m}, \frac{0.5 \cdot S_{1}}{S_{8}}\right)$

The failure modes $E$ and $F$ are related to the extreme horizontal ground deformations, respectively, in extension and in compression. These two types of deformation have to be considered separately because the tolerance of a structure to extension is generally smaller than the one to compression (especially when considering masonry buildings). It is thus desirable to adopt two different criteria, with a maximal admissible extension $\varepsilon_{t, \max }$ and a maximum admissible compression $\varepsilon_{c \text {,max }}$. The functions of performance are:
$G_{E}=\varepsilon_{t, \max }-\max \left(S_{9}, S_{11}\right)$

$G_{F}=\varepsilon_{c, \max }-\max \left(\left|S_{10}\right|,\left|S_{12}\right|\right)$

7.1 Computation of the single-mode failures probabilities

The probability of failure of each single mode is computed using Monte-Carlo simulations with $10^{5}$ samples. For each sample, the values of the output variables $S_{1}$ to $S_{12}$ are computed using the PCEs defined earlier in this paper, and the values of the functions of performance $G_{A}$ to $G_{F}$ are computed with respect to the admissible values of each failure mode. These operations are performed for normal and non-normal input variables and for the three probabilistic scenarios. All the results are gathered in Fig. 11 and show that a similar behavior exists for the six limit states. In the range $[0.1 ; 0.0001]$, one may observe that the decrease of the logarithm of the failure probability is quasilinear with the increase of the corresponding admissible threshold. This is true for the six limit states, but cannot be extrapolated for smaller values of the failure probability. Indeed, the relatively low order of the PCEs does not allow to achieve a good accuracy for failure probabilities lower than $10^{-4}$.

Figure 11 shows that considering non-normal variables considerably reduces the failure probability for a given mode. The factor of reduction can reach one or two orders of magnitude, which is very significant. The use of normal input variables may therefore be considered as a conservative assumption because it leads to much more dispersed output variables and probably tends to overestimate the failure probabilities. This result emphasizes the need of a precise description of the statistical distributions of the 

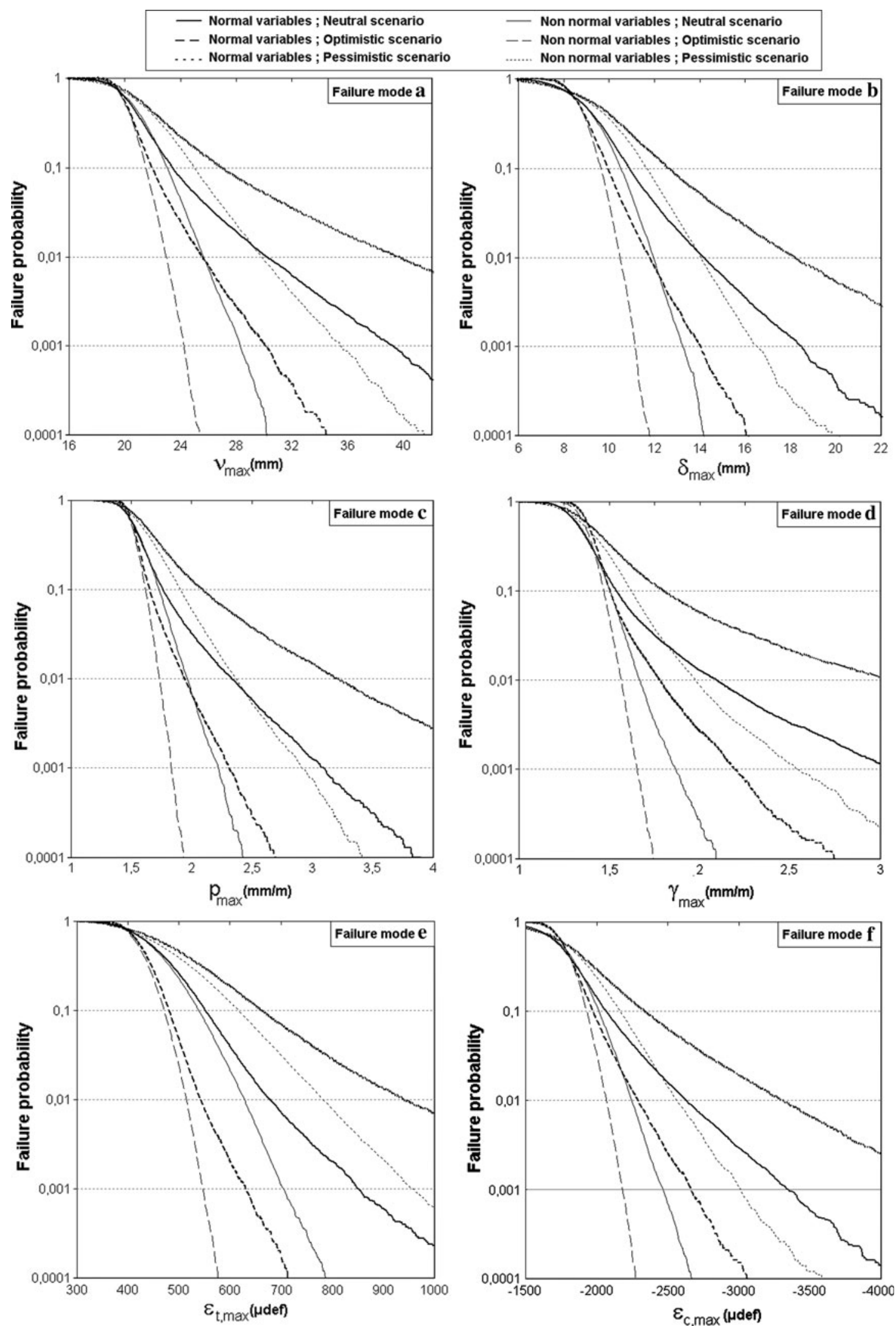

Fig. 11 Single-mode failure probabilities of the excavation with respect to the admissible values of each limit state, depending on the probabilistic scenario and type of distribution of the input variables 
Table 5 Single-mode and multi-mode probabilities of failure

\begin{tabular}{|c|c|c|c|c|c|}
\hline & \multicolumn{2}{|c|}{ Admissible values } & \multicolumn{3}{|c|}{ Probabilistic scenario } \\
\hline & Name & Value & Optimistic & Neutral & Pessimistic \\
\hline \multicolumn{6}{|c|}{ Single-mode failure probabilities } \\
\hline Mode $A$ & $v_{\max }(\mathrm{mm})$ & 30 & 0.0011 & 0.0108 & 0.0527 \\
\hline Mode $B$ & $\delta_{\max }(\mathrm{mm})$ & 15 & 0.00034 & 0.00636 & 0.0343 \\
\hline Mode $C$ & $p_{\max }(\mathrm{mm} / \mathrm{m})$ & 2.5 & 0.00032 & 0.00609 & 0.0378 \\
\hline Mode $D$ & $\gamma_{\max }(\mathrm{mm} / \mathrm{m})$ & 3 & 0.00003 & 0.00116 & 0.0107 \\
\hline Mode $E$ & $\varepsilon_{t, \max }(\mu \mathrm{def})$ & 600 & 0.0022 & 0.0410 & 0.189 \\
\hline Mode $F$ & $\varepsilon_{c, \max }(\mu \mathrm{def})$ & 2,500 & 0.00248 & 0.0154 & 0.0621 \\
\hline \multicolumn{3}{|c|}{ Maximal single-mode failure probability } & 0.00248 & 0.0410 & 0.189 \\
\hline \multicolumn{3}{|c|}{ System probability of failure (multi-mode) } & 0.00326 & 0.0454 & 0.205 \\
\hline
\end{tabular}

input variables, otherwise the results in terms of failure probabilities cannot be held for certain.

\subsection{Computation of the system failure probability}

The curves presented in Fig. 11 provide the failure probability for each mode separately. Notice that one has to define a system failure probability taking into account all the failure modes. Thus, the union of the six single-mode failures should be considered. In such a case, the failure of the system is declared when at least one of the six failure criteria is reached. Mathematically, this necessitates the use of a global performance function $G$ given by the expression:

$G=\min \left(G_{A}, G_{B}, G_{C}, G_{D}, G_{E}, G_{F}\right)$

As an example, arbitrary admissible values for the six limit states are stored in Table 5, and the global probability of failure (i.e., the probability that at least one of the criteria is not satisfied) is computed using the same MonteCarlo simulation (with $10^{5}$ samples) than previously. The failure probabilities of each single mode and the global failure probability are stored in Table 5, for normal variables and for the three probabilistic scenarios. It appears that the system failure probability is generally a bit larger than the maximum of the six single-mode failure probabilities. This is due to the fact that there is always one of the modes that is predominant on the others. As a first estimate, it may thus be acceptable to consider that the system failure probability is equal to the maximum singlemode failure probability, which prevents from running a system reliability analysis as in this section.

For example, when considering the case of a neutral probabilistic scenario, one may observe that the maximal single-mode failure probability is equal to 0.0410 (mode $E$ ), while the system failure probability is equal to 0.0454 .
The difference between these two values is much smaller than the impact of the probabilistic scenario and of the type of input variables.

\section{Conclusion}

A deterministic numerical model was developed using the commercial code FLAC $^{3 \mathrm{D}}$ in order to reproduce the main phenomena occurring around a typical slurry-shield tunnel excavation. This model made possible the computation of ground movements induced by the tunnel excavation and likely to have a negative effect on existing structures at the ground surface. To make the model computationally efficient, a summation method was used, allowing the determination of the total ground movements from the ones induced by a single excavation stage. Numerical results showed the impact of the different stages of the excavation process on the soil movements. At each excavation stage, a progressive decompression of the soil was observed about ten meters in the upstream of the tunnel face, due to the face excavation. This decompression triggered a beginning of settlement. In the neighborhood of the cutting wheel, a much larger settlement appeared because of the overcutting. Behind this "peak", a small increase in settlement related to the conicity of the shield appeared. The grout injection pressure at the tail void induced a negative settlement (i.e., a soil heave) at the end of the shield, and the grout consolidation and solidification induced a last settlement peak.

The variability of seven important input variables of the model (related to soil properties and retaining fluid pressures) was modeled mathematically using random variables, and several probabilistic scenarios were built to define the dispersion of these input variables. An efficient probabilistic method called CSRSM was used to assess the 
uncertainty propagation through the numerical model and to characterize the variability and cross-correlation of its 12 output variables. In a certain range of problems (for example when studying the impact of a tunnel excavation on existing buildings), these variables may be used directly as input random variables without using a complex deterministic model to describe the excavation process. However, one should keep in mind before using these results that they were obtained in a free-field configuration and therefore do not consider any interaction between soil and possibly existing structures. This more complex topic is left for further studies, but the present results may be used as a first approximation.

Finally, six modes of failure of the excavation were defined, depending on admissible values of the ground movements provided by the probabilistic model. Each of these movements (e.g., settlement, horizontal displacement, slope, or horizontal deformation) is likely to have a negative impact on any existing structure at the ground surface. Single-mode (i.e., considering only one failure criterion) and System (i.e., considering the union of the six failure criteria) failure probabilities were computed. It was shown that the system probability failure is generally slightly larger than the maximum failure probability of the six modes taken separately.

As for any probabilistic study in geotechnical engineering, some comments are needed about the accuracy of the results. The current knowledge about the real statistical distribution of soil parameters is rather limited. Designers who would wish to run probabilistic analyses may have difficulties in choosing the appropriate probabilistic scenario. This is a problem since such choice has a large influence on the computed failure probabilities. Despite a certain lack of accuracy in the determination of these probabilities, we believe that probabilistic studies do have some merit in geotechnical engineering for two main reasons: First, they provide an estimate of the failure probability (at least its order of magnitude) which is linked, on a rational basis, to a set of well-defined failure criterions and to the amount of uncertainty involved in the problem. Second, they make it possible to run a rigorous sensitivity study in the early stages of a project using Sobol indices (as proposed in the present paper), and thus, they allow one to know on which parameters additional information should be gathered to enhance the project safety and reliability.

\section{References}

1. AFTES (1983) La méthode convergence confinement, recommandations, Tunnels et Ouvrages Souterrains, no 59., 32 p
2. Ahmed M, Iskander M (2011) Analysis of tunnelling-induced ground movements using transparent soil models. J Geotech Geoenviron Eng 137(5):525-535

3. Augarde CE, Lyamin AV, Sloan SW (2003) Stability of an undrained plane strain heading revisited. Comput Geotech 30:419-430

4. Benmebarek S, Kastner R, Ollier C (1998) Auscultation et modélisation numérique du processus de creusement à l'aide d'un tunnelier. Geotechnique 48(6):801-818

5. Bernat S, Cambou B (1998) Soil-structure interaction in Shield Tunnelling in Soft Soil. Comput Geotech 22(3):221-242

6. Burd HJ, Houlsby GT, Augarde CE, Liu G (2000) Modelling tunnelling-induced settlements of masonry buildings. Proc Inst Civ Eng Geotech 143:17-29

7. Dias D, Kastner R, Maghazi M (1999) Three dimensional simulation of slurry shield tunneling. International Symposium on Geotechnical Aspects of Underground Construction in Soft Ground, Tokyo, Japan, 6p

8. Houmadi Y, Ahmed A, Soubra AH (2012) Probabilistic analysis of a one-dimensional soil consolidation problem. Georisk: Assess Manag Risk Eng Syst Geohazards 6(1):36-49

9. Huang SP, Liang B, Phoon KK (2009) Geotechnical probabilistic analysis by collocation-based stochastic response surface method: an Excel add-in implementation. Georisk: Assessment and Management of Risk for Engineered Systems and Geohazards $3(2): 75-86$

10. Isukapalli SS (1999) An uncertainty analysis of transport-transformation models, Ph.D. thesis, The State University of New Jersey, New Brunswick, New Jersey

11. Jenck O, Dias D (2004) Analyse tridimensionnelle en différences finies de l'interaction entre une structure en béton et le creusement d'un tunnel à faible profondeur. Geotechnique 8(54):519-528

12. Kasper T, Meschke G (2006) On the influence of face pressure, grouting pressure, and TBM design in soft ground tunnelling. Tunn Undergr Space Technol 21:160-171

13. Kielbessa S, Duddeck H (1991) Stress-strain fields at the tunneling face. Three dimensional analysis for two dimensional technical approach. Rock Mech Rock Eng 24:1203-1209

14. Klar A, Osman AS, Bolton M (2007) 2D and 3D upper bound solutions for tunnel excavation using 'elastic' flow fields. Int J Numer Anal Methods Geomech 31(12):1367-1374

15. Leca E, Dormieux L (1991) Upper and lower bound solutions for the face stability of shallow circular tunnels in frictional material. Géotechnique 40(4):581-606

16. Li D, Chen Y, Lu W, Zhou C (2011) Stochastic response surface method for reliability analysis of rock slopes involving correlated non-normal variables. Comput Geotech 38-1:58-68

17. Mao N, Al-Bittar T, Soubra AH (2012) Probabilistic analysis and design of strip foundations resting on rocks obeying Hoek-Brown failure criterion. Int J Rock Mech Min Sci 49(1):45-58

18. Maynar MJM, Rodriguez LEM (2005) Discrete numerical model for analysis of earth pressure balance tunnel excavation. J Geotech Geoenviron Eng 131(10):1234-1242

19. Migliazza M, Chiorboli M, Giani GP (2009) Comparison of analytical method, 3D finite element model with experimental subsidence measurements resulting from the extension of the Milan underground. Comput Geotech 36:113-124

20. Mollon G, Dias D, Soubra A-H (2009) Probabilistic analysis and design of circular tunnels against face stability. Int J Geomech ASCE 9(6):237-249

21. Mollon G, Dias D, Soubra A-H (2009) Probabilistic analysis of circular tunnels in homogeneous soils using response surface methodology. J Geotech Geoenv Eng ASCE 135(9):1314-1325

22. Mollon G, Dias D, Soubra A-H (2010) Face stability analysis of circular tunnels driven by a pressurized shield. J Geotech Geoenv Eng ASCE 136(1):215-229 
23. Mollon G, Dias D, Soubra A-H (2011) Rotational failure mechanisms for the face stability analysis of tunnels driven by a pressurized shield. Int $\mathbf{J}$ Numer Anal Meth Geomech. doi: 10.1002/nag.962

24. Mollon G, Dias D, Soubra A-H (2011) Probabilistic analysis of pressurized tunnels against face stability using collocation-based stochastic response surface method. J Geotech Geoenv Eng ASCE 137(4):385-397

25. Mollon G, Dias D, Soubra A-H (2011) Extension of CSRSM for the parametric study of the face stability of a pressurized tunnel. Risk Assessment and Management in Geoengineering (Georisk 2011), Atlanta, USA, 26-28 June 2011, http://www.univ-nantes. fr/soubra-ah, $8 \mathrm{p}$

26. Mroueh H (1998) Tunnels en site urbain : Modélisation numérique et interaction creusement-ouvrages existants, $\mathrm{PhD}$ Thesis, Ecole Centrale de Lille, 132p (in French)

27. Mroueh H, Shahrour I (2008) A simplified 3D model for tunnel construction using tunnel boring machines. Tunn Undergr Space Technol 23:38-45

28. Phoon KK, Huang SP (2007) Geotechnical probabilistic analysis using collocation-based stochastic response surface method. In:
Kanda, Takada and Furada (eds) Applications of statistics and probability in civil engineering. Taylor and Francis Group, London

29. Phoon K-K, Kulhawy FH (1999) Evaluation of geotechnical property variability. Can Geotech J 36:625-639

30. Potts DM, Addenbrooke TL (1996) The influence of an existing surface structure on the ground movements due to tunnelling. In: Proceedings of the international conference on geotechnical aspects of underground construction in soft grounds. Balkema, Rotterdam, The Netherlands, pp 573-578

31. Soubra AH, Mao N (2012) Probabilistic analysis of obliquely loaded strip foundations. Soils Found 52(3):524-538

32. Sudret B (2007) Global sensitivity analysis using polynomial chaos expansion. Reliab Eng Syst Saf 93:964-979

33. Vermeer PA, Ruse N, Marcher T (2002) Tunnel heading stability in drained ground. Felbau 20(6):8-18

34. Xiu D, Karniadakis GE (2002) The Wiener-Askey polynomial chaos for stochastic differential equations. J Sci Comput 24(2):619-644 